\title{
Velocity-Selection Problem for Combined Motion of Melting and Solidification Fronts
}

\author{
Efim A. Brener and D. E. Temkin \\ Institut für Festkörperforschung, Forschungszentrum Jülich, D-52425 Jülich, Germany
}

(Received 21 January 2005; published 9 May 2005)

\begin{abstract}
We discuss a free boundary problem for two moving solid-liquid interfaces that strongly interact via the diffusion field in the liquid layer between them. This problem arises in the context of liquid film migration (LFM) during the partial melting of solid alloys. In the LFM mechanism the system chooses a more efficient kinetic path which is controlled by diffusion in the liquid film, whereas the process with only one melting front would be controlled by the very slow diffusion in the mother solid phase. The relatively weak coherency strain energy is the effective driving force for LFM. As in the classical dendritic growth problems, also in this case an exact family of steady-state solutions with two parabolic fronts and an arbitrary velocity exists if capillary effects are neglected [D. E. Temkin, Acta Mater. 53, 2733 (2005)]. We develop a velocity-selection theory for this problem, including anisotropic surface tension effects.
\end{abstract}

PACS numbers: 47.54.+r, 81.10.Aj

The early observations of liquid film migration (LFM) were made during sintering in the presence of liquid phase [1] or during partial melting of alloys [2] (see [3] for a review). Nowadays LFM is a well established phenomenon of great practical importance. In LFM one crystal is melted and another one is solidified. Both solid-liquid interfaces move together with the same velocity. In the investigated alloy systems the migration velocity is of the order of $10^{-6}-10^{-5} \mathrm{~cm} / \mathrm{s}$, and it is controlled by the solute diffusion through a thin liquid layer between the two interfaces [4]. The migration velocity is much smaller than the characteristic velocity of atomic kinetics at the interfaces. Therefore, both solids should at the interfaces be locally in thermodynamic equilibrium with the liquid phase. On the other hand, these local equilibrium states should be different for the two interfaces to provide the driving force for the process. It is by now well accepted (see, for example, $[3,4])$ that the difference of the equilibrium states at the melting and solidification fronts is due to the coherency strain energy, important only at the melting front because of the sharp concentration profile ahead of the moving melting front (diffusion in the solid phase is very slow and the corresponding diffusion length is very small). The solute atoms diffuse ahead of the moving film, and the coherency strain energy in such a frontal diffusion zone arises from the solute misfit (for the schematic diagram of liquid film migration see Fig. 11 in [5]). If such a frontal diffusion zone is sufficiently small due to the very slow diffusion in the solid phase, the coherency strain energy is not relaxed due to the possible nucleation of misfit dislocations [3] (for an alternative point of view see also [6]). Thus, the equilibrium liquid composition at the melting front, which depends on the coherency strain energy and on the curvature of the front, differs from the liquid composition at the unstressed and curved solidification front. This leads to the necessary gradient of the concentration across the liquid film, and the process is controlled by the diffusion in the film.
If only the melting front existed, the melting process would be controlled by the very slow diffusion in the mother solid phase and elastic effects would be irrelevant. In the LFM mechanism the system chooses a more efficient kinetic path which is controlled by the much faster diffusion in the liquid film. However, in this case the relatively weak coherency strain energy is involved as an effective driving force for this process. In this respect the LFM mechanism is similar to other well-known phenomena such as diffusion induced grain boundary migration and cellular precipitation [3]. In these processes a relatively fast diffusion along the grain boundaries controls the kinetics, and the coherency strain energy also plays a controlling role.

Thus, a theoretical description of LFM requires the solution of a free boundary problem for two combined moving solid-liquid interfaces with a liquid film in between. In Ref. [7] this problem was considered for simplified boundary conditions: the temperature and the chemical composition along each interface were kept constant. Their values are different for the melting and solidification fronts and differ from those far from the migrating liquid film. This means that any capillary, kinetic, and crystallographic effects at the interfaces were neglected. It was found that under these simplified boundary conditions two cofocal parabolic fronts can move together with the same velocity. The situation is rather similar to a steady-state motion of one parabolic solidification front into a supercooled melt $[8,9]$ or one parabolic melting front into a superheated solid. In this approximation the Peclet numbers were found, but the steady-state velocity remained undetermined at that stage. Thus, the problem of velocity-selection arises.

Solvability theory has been very successful in predicting certain properties of pattern selecting in dendritic growth and a number of related phenomena (see, for example, [10-12]). In the two-dimensional dendritic case, the basic approach is as follows. One attempts to model the dendritic 
tip by a needle crystal, that is, a shape-preserving steadystate growth shape which is a solution of the equation of motion governing diffusion in the neighborhood of a solidification front. This needle crystal is assumed to be close in shape to the parabolic Ivantsov solution. If anisotropic capillary effects are included, a single dynamically stable solution is found for any external growth conditions. This theory has been extended to the three-dimensional case $[13,14]$. We note that capillarity is a singular perturbation and the anisotropy of the surface energy is a prerequisite for the existence of the solution.

The main purpose of this Letter is to develop a velocityselection theory for LFM, including in the consideration anisotropic surface tension effects. We note that this is not just a routine extension of the existing theory because the diffusion interaction between two interfaces changes the problem substantially.

We discuss the two-dimensional problem of the steadystate motion of a thin liquid film during the process of isothermal melting of a binary alloy, see Fig. 1. We assume that the diffusion in the solid phases is very slow and the concentration $c$ in the liquid film obeys the Laplace equation. We introduce the normalized concentration $C=(c-$ $\left.c_{L}\right) /\left(c_{L}-c_{S}\right)$ with $c_{L}$ and $c_{S}$ being the liquidus and solidus concentrations of the equilibrium phase diagram at a given temperature. Then the equilibrium concentration and the mass balance conditions at the solidification front read

$$
C=d_{2} K_{2}, \quad V_{n}=-D \partial C / \partial \mathbf{n} .
$$

At the melting front the equilibrium concentration is changed by the presence of the elastic coherency strain energy [3] and also the diffusional flux changes because in the solid ahead of the melting front the concentration is $c_{0}$ which is different from $c_{S}$ :

$$
C=-b \Delta^{2}-d_{1} K_{1}, \quad V_{n}(1-\Delta)=-D \partial C / \partial \mathbf{n} .
$$

Here $V_{n}$ is the normal velocity, $D$ is the diffusion coefficient in the liquid film, $K$ is the curvature assumed to be

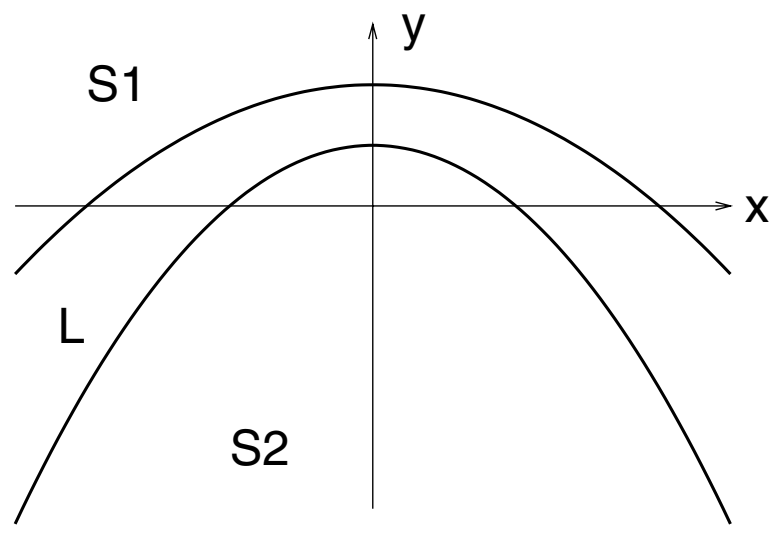

FIG. 1. Schematic presentation of two moving nearly parabolic fronts; $S 1$ and $S 2$ are the melting and growing solids, and $L$ is the liquid film. negative for the interfaces in Fig. $1, \Delta=\left(c_{0}-c_{S}\right) /\left(c_{L}-\right.$ $\left.c_{S}\right)$ is the dimensionless driving force, $b=$ $Y \Omega(d a / d c)^{2} / a^{2} f_{L}^{\prime \prime}$ is the dimensionless constant which describes the coherency strain energy [4], $\Omega$ is the atomic volume, $Y$ is the bulk elastic modulus, $a$ is the atomic constant, $f_{L}(c)$ is the free energy of the liquid phase per atom, $f_{L}^{\prime \prime}$ is the second derivative of $f_{L}(c)$ at $c=c_{L}, d_{i}$ are the anisotropic chemical capillary lengths:

$$
d_{i}(\theta)=d_{0}\left[1-\alpha \cos 4\left(\theta-\theta_{i}\right)\right], \quad d_{0}=\gamma \Omega / f_{L}^{\prime \prime}\left(c_{L}-c_{S}\right)^{2}
$$

with the isotropic part of the surface energy $\gamma$ and the anisotropy parameter $\alpha \ll 1, \theta$ is the angle between the normal to the interface $\mathbf{n}$ and the direction of motion, and $\theta_{i}$ is the direction of the minimum of $d_{i}(\theta)$ for each of the interfaces.

We measure all lengths in the units of the radius of curvature of the Ivantsov parabolic solidification front, $R_{2}$. Introducing a parabolic coordinates system,

$$
y=\left(\eta^{2}-\beta^{2}\right) / 2, \quad x=\eta \beta,
$$

we look for a solution of the Laplace equation for the concentration field $C(\eta, \beta)$ in the liquid phase with the following boundary conditions:

$$
\begin{aligned}
& C\left[\eta=\eta_{2}(\beta), \beta\right]=d_{2}(\beta) K_{2}(\beta), \\
& C\left[\eta=\eta_{1}(\beta), \beta\right]=-b \Delta^{2}-d_{1}(\beta) K_{1}(\beta) .
\end{aligned}
$$

The mass balance conditions at the interfaces are

$$
\begin{aligned}
2 P\left[\eta_{2}(\beta)+\beta \eta_{2}^{\prime}(\beta)\right] & =-\left(\partial C / \partial \eta-\eta_{2}^{\prime} \partial C / \partial \beta\right), \\
2 P(1-\Delta)\left[\eta_{1}(\beta)+\beta \eta_{1}^{\prime}(\beta)\right] & =-\left(\partial C / \partial \eta-\eta_{1}^{\prime} \partial C / \partial \beta\right),
\end{aligned}
$$

where the Peclet number is $P=V R_{2} / 2 D$ and $V$ is the steady-state velocity in the $y$ direction. We present the two moving interfaces in the form

$$
\eta_{2}=1+\epsilon_{2}(\beta), \quad \eta_{1}=\eta_{0}+\epsilon_{1}(\beta)
$$

and the concentration field in the form

$$
C(\eta, \beta)=C_{0}(\eta)+u(\eta, \beta),
$$

where $C_{0}(\eta)=-b \Delta^{2}(\eta-1) /\left(\eta_{0}-1\right)$ is the solution without surface tension $\left(u=\epsilon_{1}=\epsilon_{2}=0\right)$ which satisfies the boundary conditions: $C_{0}(1)=0$ and $C_{0}\left(\eta_{0}\right)=$ $-b \Delta^{2}$. The balance equations at the interfaces, $2 P=$ $2 P(1-\Delta) \eta_{0}=-\partial C_{0} / \partial \eta$, lead to the relations for the Peclet number and $\eta_{0}$ :

$$
P=\frac{V R_{2}}{2 D}=\frac{b \Delta^{2}}{2\left(\eta_{0}-1\right)}, \quad \eta_{0}=\sqrt{\frac{R_{1}}{R_{2}}}=\frac{1}{1-\Delta} .
$$

These relations can also be obtained in the proper limit of Eqs. (27) and (28) of Ref. [7] and give the expressions for the radii of curvature for the two interfaces, $R_{1}$ and $R_{2}$, for 
a given velocity $V$ which remains undetermined at this stage.

The surface tension plays a crucial role in the velocityselection problem. In order to find the small corrections to these solutions due to the small (but singular) surface tension effects, we should first find the small correction $u$ to the diffusional field in the linear approximation with respect to $\epsilon$ and $d K$ (see, for example, [12]). In this approximation, the field $u$ satisfies the Laplace equation and the boundary conditions:

$$
\begin{gathered}
u(\eta=1, \beta)=\Psi_{2}(\beta)=2 P \epsilon_{2}+d_{2} K_{2}, \\
u\left(\eta=\eta_{0}, \beta\right)=\Psi_{1}(\beta)=2 P \epsilon_{1}-d_{1} K_{1} .
\end{gathered}
$$

The balance equations read

$$
\begin{aligned}
2 P\left(\epsilon_{2}+\beta \epsilon_{2}^{\prime}\right) & =-\partial u /\left.\partial \eta\right|_{\eta=1}, \\
2 P(1-\Delta)\left(\epsilon_{1}+\beta \epsilon_{1}^{\prime}\right) & =-\partial u /\left.\partial \eta\right|_{\eta=\eta_{0}} .
\end{aligned}
$$

The Laplace equation with the boundary conditions, Eq. (10), is easily solved by Fourier transform, with respect to the variable $\beta$, which we define as

$$
\overline{g(\lambda)}=\frac{1}{\sqrt{2 \pi}} \int_{-\infty}^{\infty} d \beta \exp (-i \lambda \beta) g(\beta) .
$$

Then,

$$
\overline{u(\eta, \lambda)}=\frac{\bar{\Psi}_{1} \sinh [\lambda(\eta-1)]}{\sinh \left[\lambda\left(\eta_{0}-1\right)\right]}-\frac{\bar{\Psi}_{2} \sinh \left[\lambda\left(\eta-\eta_{0}\right)\right]}{\sinh \left[\lambda\left(\eta_{0}-1\right)\right]} .
$$

Finally, Eqs. (11) read

$$
\begin{aligned}
& 2 P \frac{d \bar{\epsilon}_{2}}{d \lambda}=\frac{\bar{\Psi}_{1}}{\sinh \left[\lambda\left(\eta_{0}-1\right)\right]}-\frac{\bar{\Psi}_{2} \cosh \left[\lambda\left(\eta_{0}-1\right)\right]}{\sinh \left[\lambda\left(\eta_{0}-1\right)\right]}, \\
& 2 P(1-\Delta) \frac{d \bar{\epsilon}_{2}}{d \lambda}=\frac{\bar{\Psi}_{1} \cosh \left[\lambda\left(\eta_{0}-1\right)\right]}{\sinh \left[\lambda\left(\eta_{0}-1\right)\right]}-\frac{\bar{\Psi}_{2}}{\sinh \left[\lambda\left(\eta_{0}-1\right)\right]}
\end{aligned}
$$

Eliminating $\bar{\epsilon}_{1}$ from the first of these two equations,

$$
\begin{aligned}
2 P \bar{\epsilon}_{1}= & 2 P\left(\bar{\epsilon}_{2}\right)^{\prime} \sinh \left[\lambda\left(\eta_{0}-1\right)\right]+2 P \bar{\epsilon}_{2} \cosh \left[\lambda\left(\eta_{0}-1\right)\right] \\
& +\overline{d_{1} K_{1}}+\overline{d_{2} K_{2}} \cosh \left[\lambda\left(\eta_{0}-1\right)\right],
\end{aligned}
$$

we find from the second one,

$$
\begin{aligned}
2 P\left(\bar{\epsilon}_{2}\right)^{\prime \prime}-2 P \bar{\epsilon}_{2}= & \overline{d_{2} K_{2}}-\left[\frac{\left(\overline{d_{1} K_{1}}\right)^{\prime}}{\sinh \left[\lambda\left(\eta_{0}-1\right)\right]}\right. \\
& \left.+\left(\overline{d_{2} K_{2}}\right)^{\prime} \frac{\cosh \left[\lambda\left(\eta_{0}-1\right)\right]}{\sinh \left[\lambda\left(\eta_{0}-1\right)\right]}\right] .
\end{aligned}
$$

Here the prime denotes derivatives with respect to $\lambda$.

These equations are convenient for further analysis. We are interested in the motion of the thin film, which means that $\left(\eta_{0}-1\right) \approx \Delta \ll 1$. In this case we can expand the hyperbolic functions for small values of their argument almost everywhere. This leads to $\epsilon_{1} \approx \epsilon_{2}, K_{1} \approx K_{2}$, and then, from Eq. (15), we find in direct space

$$
\frac{d}{d \beta}\left[\left(1+\beta^{2}\right) \epsilon_{2}\right]=\frac{d_{1}+d_{2}}{2 P \Delta} \beta K_{2} .
$$

A few remarks are in order. First of all, this is somewhat the expected result because for the thin films the resulting equation should be local and, in principal, can be derived in the framework of boundary layer techniques. However, the used approximation for small arguments of hyperbolic functions breaks down in the small vicinity (of the order of $\Delta$ ) near the singular points in the complex plane, $\beta= \pm$ $i$. We discuss this point later. Finally, we can return to Cartesian coordinates because the expression for the curvature is much simpler in this representation. In the linear approximation $\left(1+\beta^{2}\right) \epsilon_{2} \approx \zeta_{2}(x)$ where $\zeta_{2}(x)$ is the correction to the parabolic solution in the Cartesian coordinates. Then Eq. (16) reads

$$
\frac{d \zeta_{2}}{d x}=\frac{d_{1}+d_{2}}{2 P \Delta} x K_{2} .
$$

One finds the first regular corrections to the parabolic shape by replacing $d_{1}=d_{2}=d_{0}$ and $K_{2}=-1 /\left[R_{2}(1+\right.$ $\left.x^{2}\right)^{3 / 2}$ ] for the parabola:

$$
\zeta_{2}(x)=\sigma /\left[\Delta\left(1+x^{2}\right)^{1 / 2}\right], \quad \Delta \ll 1,
$$

where $\sigma=d_{0} /\left(P R_{2}\right)$ is the usual stability parameter which appears in such problems.

In the vicinity of the point $x=-i$ where the curvature becomes singular and anisotropy, Eq. (3), becomes important we rescale the variables [12]:

$$
x=-i(1-\sqrt{\alpha} z), \quad \zeta_{2}=\alpha F, \quad \tau=z+d F / d z,
$$

with a small anisotropy parameter, $\alpha \ll 1$. Then Eq. (17) reads

$$
\frac{d F}{d z}=-\frac{\sigma}{\Delta \alpha^{5 / 4}} A(\tau) \frac{1+d^{2} F / d z^{2}}{(2 \tau)^{3 / 2}},
$$

where the anisotropy factor is $A(\tau)=1-\left[\exp \left(-4 i \theta_{1}\right)+\right.$ $\left.\exp \left(-4 i \theta_{2}\right)\right] / \tau^{2}$. This equation is similar to the limit of the kinetic dendrite in Eq. (9.1) of Ref. [12]. This similarity is purely formal because the real interface kinetic effects are not included in our description. Instead, the strong diffusion interaction leads to such a selection problem. The same arguments as in [12] give the selection condition

$$
\sigma=\sigma^{\star} \sim \Delta \alpha^{5 / 4}, \quad \Delta \ll \sqrt{\alpha} .
$$

Moreover, the selected growth direction lies between the directions of the minima of $d_{1}(\theta)$ and $d_{2}(\theta)$ for the two interfaces, $\theta_{1}=-\theta_{2}$, because the coefficient appearing in $A(\tau)$ must be real [12]. The restriction condition in Eq. (21), $\Delta \ll \sqrt{\alpha}$, follows from the fact that the characteristic scale $1 / \lambda \sim \sqrt{\alpha}$ and $\lambda \Delta$ must be small.

While the regular correction given by Eq. (18) is valid for $\Delta \ll 1$, the restriction for the validity of inner Eq. (20) 
and for the scaling relation, Eq. (21), is much stronger, $\Delta \ll \sqrt{\alpha} \ll 1$. In the opposite limit, $\Delta \gg \sqrt{\alpha}$, we can derive another local inner equation where the important scale $|x+i| \sim \sqrt{\alpha}$ is much smaller than $\Delta$. The argument of the hyperbolic functions becomes large, and we can neglect the term $K_{1}$ in Eq. (15). Then, in the vicinity of the singular point $x=-i$ in the complex plane, this equation reads

$$
\zeta_{2}(x)=-d_{2} K_{2} / P
$$

which is the usual inner equation for a single dendrite in the one-sided model [12]. With the same rescaling as in Eq. (19), we find

$$
F=\frac{\sigma}{\alpha^{7 / 4}} A(\tau) \frac{1+d^{2} F / d z^{2}}{(2 \tau)^{3 / 2}},
$$

where the anisotropy factor is $A(\tau)=1-$ $2 \exp \left(-4 i \theta_{2}\right) / \tau^{2}$. This corresponds to the selection condition

$$
\sigma=\sigma^{\star} \sim \alpha^{7 / 4}, \quad \Delta \gg \sqrt{\alpha},
$$

and the selected growth direction corresponds to the minimum of $d_{2}(\theta), \theta_{2}=0$. Whereas Eq. (23) and the selection condition Eq. (24) are valid not only for small $\Delta$ [12], the regular correction in the form of Eq. (18) requires $\Delta \ll 1$.

The selection conditions, Eqs. (21) and (24), together with the relations for the Peclet numbers, Eq. (9), solve the posed problem of pattern formation for two combined nearly parabolic fronts:

$$
V=\frac{2 D}{d_{0}} P^{2}(\Delta) \sigma^{\star}
$$

and

$$
R_{2}=d_{0} /\left[P(\Delta) \sigma^{\star}\right], \quad R_{1}=R_{2} /(1-\Delta)^{2} .
$$

While these results are formally similar to the free dendritic case, the selected stability parameter $\sigma^{\star}$ scales as $\alpha^{7 / 4}$ only for $\Delta \gg \sqrt{\alpha}$. Otherwise, the other scaling relation, Eq. (21), holds. In principle, it could be conceivable that the strong diffusional interaction between the front leads to the selection even without anisotropy. However, our analysis of the inner equation does not support this hypothesis. The Peclet number is $P(\Delta) \sim b \Delta$ which reflects the fact that the coherency strain energy plays a crucial role in the LFM mechanism. This parameter $b$ is usually small but the melting process by the LFM mechanism is controlled by the fast diffusion in the liquid, whereas the process with only one melting front would be controlled by the very slow diffusion in the mother solid phase. As we already noted, with the help of the LFM mechanism the system chooses a more efficient kinetic path to relax to the equilibrium state.

Finally, we estimate the velocity $V$ from Eq. (25) and the thickness of the film $R_{2} \Delta$ from Eq. (26) using character- istic values of the parameters: $D \sim 10^{-5} \mathrm{~cm}^{2} / \mathrm{s}, d_{0} \sim$ $10^{-7} \mathrm{~cm}, b \sim 0.05, \Delta \sim 0.05$, and $\sigma^{\star} \sim 10^{-2}$. This leads to $V \sim 10^{-5} \mathrm{~cm} / \mathrm{s}$ and $R_{2} \Delta \sim 10^{-4} \mathrm{~cm}$, which qualitatively agree with typical values in LFM experiments.

In conclusion, we developed a selection theory for the process of liquid film migration where the strong diffusion interaction between melting and solidification fronts plays a crucial role. This process is very important in practical applications, in particular, during sintering in the presence of the liquid phase $[1,3]$. However, despite its practical importance, experimental investigations of this process are far from the level of accuracy of the model experiments in classical dendritic growth. Perhaps, this is due to the fact that practically important alloys are not very suitable for accurate testing of the pattern formation process, while the most detailed and elegant experiments in dendritic growth were performed with model transparent materials. The other reason could be that the selection theory developed for dendritic growth was not applied to LFM so far. Our approach extends selection theory developed for dendritic growth to LFM, and we hope that our results will stimulate further theoretical and experimental investigations in this very interesting field. From the theoretical side it would be a challenge to attack this problem by a direct numerical approach, for example, by means of the boundary integral method or by means of the phase-field model.

We acknowledge the support by the Deutsche Forschungsgemeinschaft under Project No. SPP 1120.

[1] D. N. Yoon and W. J. Hupmann, Acta Metall. 27, 973 (1979).

[2] T. Muschik, W. A. Kaysser, and T. Hehenkamp, Acta Metall. 37, 603 (1989).

[3] D. N. Yoon, Int. Mater. Rev. 40, 149 (1995).

[4] D. N. Yoon, J. W. Cahn, C. A. Handwerker, J.E. Blendell, and Y.J. Baik, in Interface Migration and Control of Microstrucutres (American Society for Metals, Metals Park, OH, 1985), pp. 19-31.

[5] W. A. G. McPhee, G. B. Schaffer, and J. Drennan, Acta Mater. 51, 3701 (2003).

[6] J. S. Kirkaldy, Acta Mater. 46, 5127 (1998).

[7] D. E. Temkin, Acta Mater. 53, 2733 (2005).

[8] G.P. Ivantsov, Dokl. Akad. Nauk SSSR 58, 567 (1947).[Sov. Phys. Dokl. 58, 567 (1947)].

[9] G.P. Ivantsov, Dokl. Akad. Nauk SSSR 83, 573 (1952).[Sov. Phys. Dokl. 83, 573 (1952)].

[10] Y. Saito, Statistical Physics of Crystal Growth (World Scientific Publishing, Singapore, 1996).

[11] D. Kessler, J. Koplik, and H. Levine, Adv. Phys. 37, 255 (1988).

[12] E. A. Brener and V.I. Mel'nikov, Adv. Phys. 40, 53 (1991).

[13] M. Ben Amar and E. Brener, Phys. Rev. Lett. 71, 589 (1993).

[14] E. Brener, Phys. Rev. Lett. 71, 3653 (1993). 